\title{
Influence of cutting conditions on the cutting performance of TiAl6V4
}

\author{
GuiGen $\mathrm{Ye}^{1,2, a}$, ShiFeng Xue ${ }^{1}$, XingHua Tong ${ }^{1}$ and LanHong Dai ${ }^{2, b}$ \\ ${ }^{1}$ Department of Engineering Mechanics, Petroleum University of China, Shan Dong 257061, China \\ ${ }^{2}$ State Key Laboratory of Nonlinear Mechanics, Institute of Mechanics, Chinese Academy of \\ Sciences, Beijing 100190, China \\ aYegg@Inm.imech.ac.cn, 'Ihdai@Inm.imech.ac.cn (corresponding author)
}

Keywords: Chip morphology, Size effect, Cutting temperature, TiAl6V4.

\begin{abstract}
The FEM simulation was developed to investigate the influences of cutting speed, uncut chip thickness and tool rake angle on the cutting performance of TiAl6V4. The FEM simulation was validated using experimental results and good agreement was obtained. The simulation results indicate that the transition from continuous to saw-tooth chip formation is favored by increases in cutting speed and uncut chip thickness and a decrease in tool rake angle. The existence of Salomon's assumption was further discussed, which shows that the Salomon's assumption is not valid for the tool-chip interface temperature, but could be true for the surface temperature of the workpiece. In addition, decreasing the uncut chip thickness leads to an increase of the specific cutting energy, which could be primarily caused by an increase in the shear strength of the workpiece material due to a decrease in the PSZ temperature.
\end{abstract}

\section{Introduction}

Metal cutting is the common manufacturing process used to remove unwanted materials from a workpiece and obtain designed geometrical dimensions [1]. Because of its great economic and technical importance, a great number of researches have been carried out in order to optimize the machining process in terms of improving quality, increasing productivity and lowering cost.

Titanium alloys are very interesting materials for industrial applications because of their high strength-on-weight ratio, combined with an excellent corrosion resistance at high temperatures. Despite these features, the utilization of titanium alloys is still limited due to their poor machinability. In fact, the titanium low thermal conductivity hinders the evacuation of the heat generated during the cutting process resulting in a temperature rise of the workpiece[2]. Thus the mechanism of chip formation when machining titanium alloys differs from most other materials. Specifically, it has been found that the serrated or saw-tooth chips are prone to be formed over a wide range of cutting speed. The saw-tooth chip formation ties up with decreased tool life, degradation of the workpiece surface finish and less accuracy in the machined part [3]. Therefore, investigating the influence of cutting conditions on the machining performance for titanium alloys is of significant importance. It could lead to improvements in tool life, component surface finish and dimensional control which, in turn, would lead to improvements to productivity.

In this work, the numerical simulation, which was validated by the orthogonal cutting experiments, was carried out to investigate the influence of cutting speed, uncut chip thickness and tool rake angle on the chip morphology, cutting force and temperature during machining TiAl6V4 alloy. This could assist in the optimization of the cutting conditions for TiAl6V4.

\section{The FEM Simulation}

In this work, the finite element model (FEM) was developed to simulate the orthogonal cutting process. The FEM is a fully coupled thermal-mechanical model, developed in the commercially available software, ABAQUS/Explicit. For the simulations, the workpiece is discretized using 4-node bilinear elements with reduced integration. As for the tool, it is treated here for simplicity as an analytical rigid body. 
To model the thermal-viscoplastic behavior of the workpiece material, the software uses a constitutive equation, the Johnson-Cook law, which can be represented by the following formula:

$$
\sigma=\left(A+B \varepsilon^{n}\right)\left(1+C \ln \frac{\dot{\varepsilon}}{\dot{\varepsilon}_{0}}\right)\left(1-\left(\frac{T-T_{0}}{T_{m}-T_{0}}\right)^{m}\right)
$$

Table 1 The material parameters of TiAl6V4.

\begin{tabular}{cccccccc}
\hline$A(\mathrm{MPa})$ & $B(\mathrm{MPa})$ & $C$ & $n$ & $m$ & $T_{0}(\mathrm{~K})$ & $T_{m}(\mathrm{~K})$ & $\dot{\varepsilon}_{0}$ \\
\hline 789 & 529 & 0.028 & 0.28 & 1 & 293 & 1873 & $1 \mathrm{E}-5$ \\
\hline
\end{tabular}

The material parameters of TiAl6V4 are listed in Table 1 [4].

The extended Coulomb friction model is chosen in this work to model the tool-chip interaction, and the friction coefficient is set to be 0.3 as a constant [5].

The separation of the chip from the workpiece is modeled with the help of a failure zone. Up to failure, this zone behaves according to the current Johnson-Cook-based model described in Eq. (1). The failure of this zone takes place at a critical value $\tilde{\varepsilon}_{p}$ of the accumulated equivalent inelastic deformation, and hence the chip separates from the surrounding workpiece in a defined distance from the tool tip. In this work, the $\tilde{\varepsilon}_{p}$ is set to a value of 2 [4].

\section{The FEM Simulation Verification}

In order to validate the FEM simulation, orthogonal cutting tests were undertaken using a TiAl6V4 alloy with uncoated P10 carbide tool. The workpiece was in the form of a $100 \mathrm{~mm}$ diameter disc, 2 $\mathrm{mm}$ in width. New cutting inserts were used for each test to limit the tool wear. The test conditions are listed in Table 2.

Table 2 The test conditions.

\begin{tabular}{cccc}
\hline Tool rake angle & Clearance angle & Uncut chip thickness & Cutting speed \\
\hline $0^{0}$ & $7^{0}$ & $0.1 \mathrm{~mm}$ & $3-90 \mathrm{~m} / \mathrm{min}$ \\
\hline
\end{tabular}

After cutting, the chips have been collected and embedded into resin. The lateral section was mechanically polished and then etched. The microstructures of the chips obtained at different cutting speeds are shown in Fig.1.

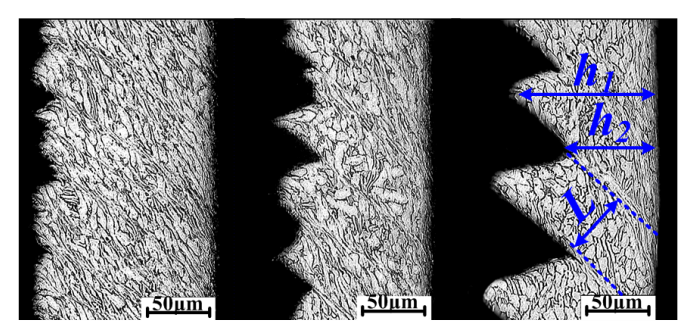

Fig.1 The microstructure of chip formation at cutting speed of: (a) $3 \mathrm{~m} / \mathrm{min}$, (b) $30 \mathrm{~m} / \mathrm{min}$, (c) $90 \mathrm{~m} / \mathrm{min}$.

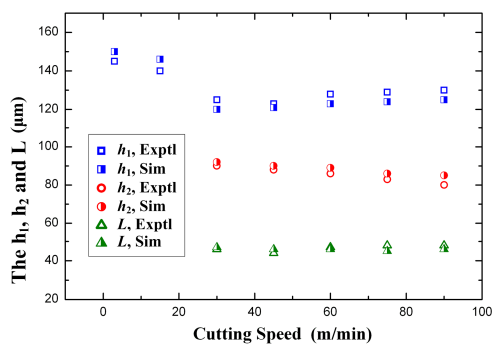

Fig.2 Comparison of experimental and simulated chip morphology. Where $h_{1}, h_{2}$ and $L$ are marked in Fig. 1c

The corresponding FEM simulations were carried out by using the cutting parameters listed in Table 2. Fig. 2 gives the comparison between the simulated chip morphologies with the experimental ones. As seen from Fig. 2, good agreement is obtained between the simulated and experimental results. For each cutting speed, the simulated results of the maximum $\left(h_{1}\right)$ and minimum $\left(h_{2}\right)$ chip height as well as the tooth spacing $(L)$ are in accordance with the experimental ones. Therefore, the finite element model is considered to be satisfactorily validated and is used in the following section to analyze the influence of cutting conditions on the chip morphology, cutting force and temperature. 


\section{Influence of cutting parameters on the cutting performance}

Influence of cutting speed. In order to investigate the influence of cutting speed on the cutting performance, the uncut chip thickness and tool rake angle are set to be $0.1 \mathrm{~mm}$ and $0^{0}$ respectively as a constant. The cutting speed varies from 3 to $600 \mathrm{~m} / \mathrm{min}$.

The simulation results for different cutting speeds show that, with increasing the cutting speed, the chip morphology transfers from continuous to saw-tooth-like, as shown in Figs.3.

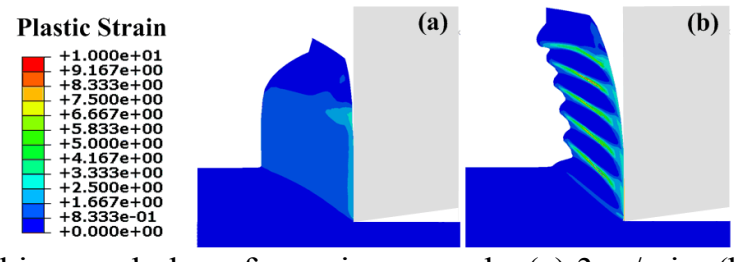

(c)

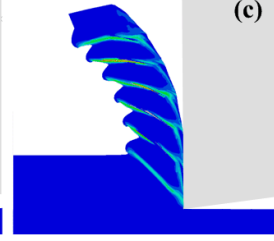

Fig. 3 Chip morphology for various speeds: (a) $3 \mathrm{~m} / \mathrm{min}$, (b) $30 \mathrm{~m} / \mathrm{min}$, (c) $90 \mathrm{~m} / \mathrm{min}$.

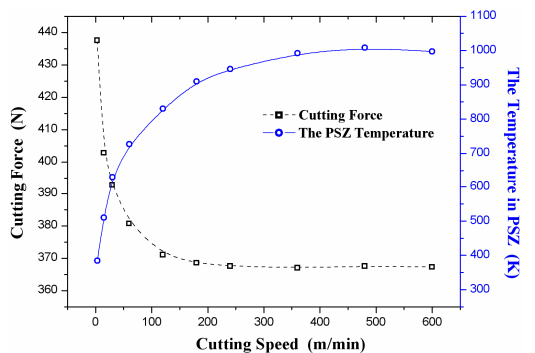

Fig.4 Cutting force and PSZ temperature plotted with cutting speed.

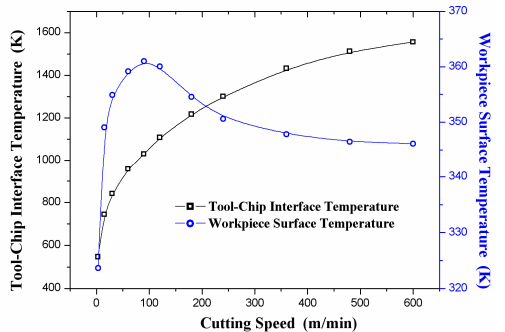

Fig.5 Tool-chip interface temperature and workpiece surface temperature plotted with cutting speed.

Fig. 4 shows the variations of the cutting force and the primary shear zone (PSZ) temperature with respect to the cutting speed. Increasing the cutting speed will accelerate the heat generation in the PSZ, and thus the temperature in the PSZ increases, as seen the solid line in Fig.4. Furthermore, the temperature rise decreases the shear strength of work material due thermal softening, which would reduce the plastic work and hence lead to a reduction of the cutting force, see the dashed line in Fig.4.

High speed machining (HSM) has been used worldwide for its excellent advantages. The concept of HSM was introduced by Salomon [6], which assumed that the cutting temperature decreases with increasing the cutting speed after it attains a peak for a certain critical value. Salomon's assumption is one of the most widely considered and quoted hypothesises in the world. However, it is still controversial about whether the Salomon's assumption is valid. As shown in Fig. 5, the tool-chip interface temperature increases with speed, with no reduction at higher speeds, and the workpiece surface temperature decreases with increase in the speed after it attains a peak. This tend demonstrates that, the Salomon's assumption is not valid for the tool-chip interface temperature, but could be true for the workpiece temperature. According to Shaw [7], about $90 \%$ of the heat generated goes into the chip, and only 5\% into the workpiece. As the cutting speed increases there is less time available for the heat generated in PSZ to conduct into the workpiece. So the heat is more effectively carried away with the chip at higher cutting speed, therefore the tool-chip temperature rises and the workpiec temperature stays relatively cool. Thus, there can be significant differences between the tool-chip contact temperature and the workpiece surface temperature. And hence, the point at which the cutting temperature is defined is important to study the Salomon's assumption.

Influence of uncut chip thickness. In order to investigate the influence of cutting speed on the cutting performance, the cutting speed and tool rake angle are set be $60 \mathrm{~m} / \mathrm{min}$ and $0^{0}$ respectively, and the uncut chip thickness varies from 0.01 to $0.15 \mathrm{~mm}$.

The simulated chip morphologies for different uncut chip thickness are shown in Fig.6. Form Fig.6 it can be found that the chip morphology transfers from continuous to saw-tooth-like as the uncut chip thickness increasing. 
Fig.7a gives the curves of the PSZ temperature, tool-chip interface temperature and workpiece surface temperature versus the uncut chip thickness. It can be noticed that with increasing the uncut chip thickness, the tool-chip interface and workpiece surface temperature basically remain stable. However, increasing the uncut chip thickness leads to a continuous increase of the PSZ temperature.

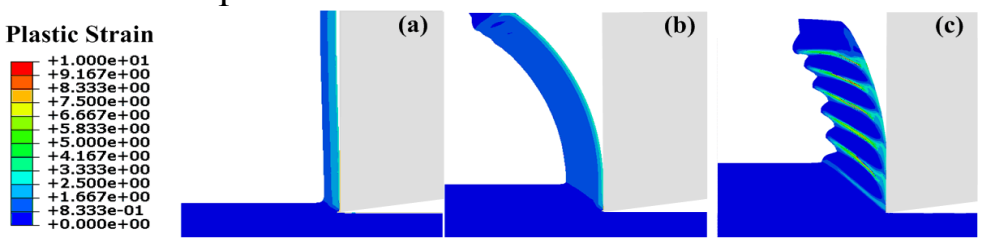

Fig.6 Chip morphology for various uncut chip thickness: (a) $0.015 \mathrm{~mm}$, (b) $0.05 \mathrm{~m}$, (c) $0.1 \mathrm{~mm}$.

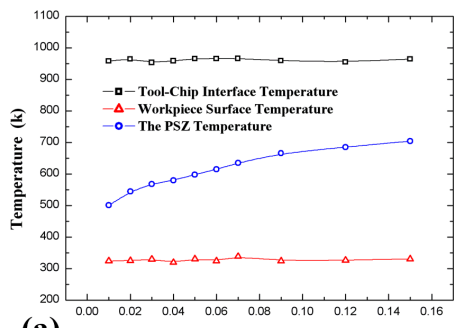

(a)
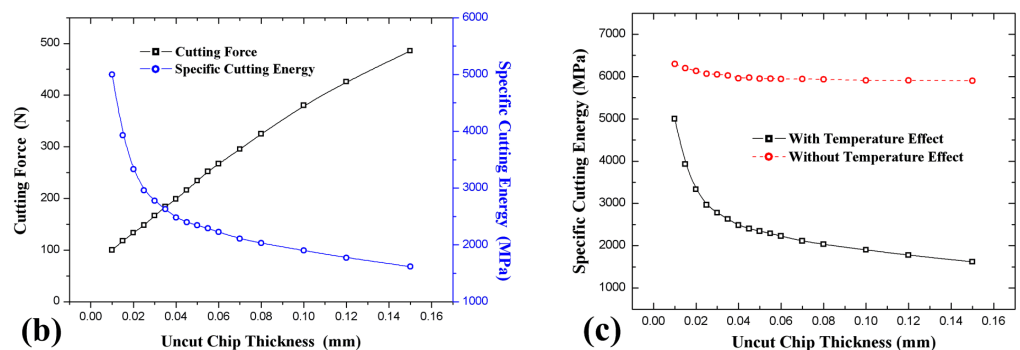

(c)

Fig.7 (a) Temperatures versus uncut chip thickness, (b) Cutting force and specific cutting energy versus uncut chip thickness, and (c) The specific cutting energy versus uncut chip thickness with and without the temperature effect.

The influences of uncut chip thickness on the cutting force and the specific cutting energy are shown in Fig. 7b. Form Fig. 7b it can be easily found that, increasing the uncut chip thickness increases the cutting force, since more energy is consumed to remove the additional work material. Moreover, from Fig.7b it also can be found that the specific cutting energy, the amount of energy required for removing unit volume of material, increases nonlinearly when the uncut chip thickness decreases from a 150 microns to 10 microns. This scaling phenomenon is also termed the size effect. During past 50 years, extensive work in this area has been carried out to explanation this phenomenon. However, the cause for the size effect is still unclear.

As shown in Fig. 7a, decreasing the uncut chip thickness decreases the temperature in the PSZ, which would lead to an increase in the shear strength of the workpiece material and hence increase the specific cutting energy. Therefore, the occurrence of size effect phenomenon could be attributed to a drop in the PSZ temperature with decrease in the uncut chip thickness. In order to confirm this point, another set of simulations was carried out, where the temperature term in Eq. (1) was not considered (the thermal softening coefficient $m$ was set to be zero). Thus the work material deforms without any thermal softening. Figure 7c shows a plot of the specific cutting energy versus uncut chip thickness with and without the temperature effect. It can be seen that, when the temperature effect is not considered, the size effect phenomenon vanishes. It is clear from Fig.7 that the workpiece thermal softening effect is a dominant mechanism responsible for the size effect. And thus, the seize effect could be primarily caused by an increase in the shear strength of the workpiece material due to a decrease in the PSZ temperature.

Influence of tool rake angle. In order to investigate the influence of cutting speed on the cutting performance, the uncut chip thickness and cutting speed are set be $0.1 \mathrm{~mm}$ and $60 \mathrm{~m} / \mathrm{min}$ respectively, and the tool rake angle varies from $-20^{\circ}$ to $20^{\circ}$.

The simulated chip morphologies for different tool rake angle are shown in Fig.8. It can be seen that, the saw-tooth chip formation is favored by a decrease in tool rake angle.
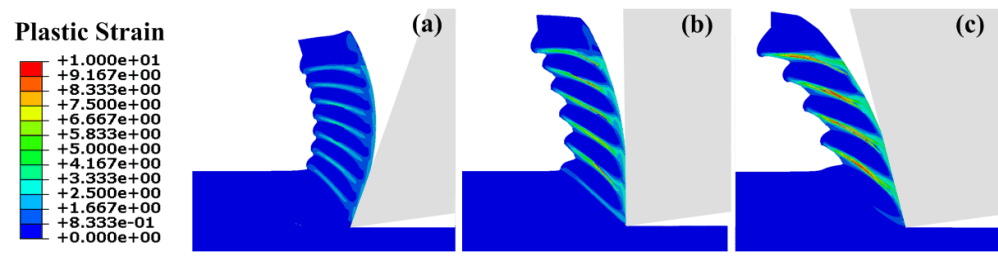

Fig.8 Chip morphology for various tool rake angle: (a) $20^{\circ}$, (b) $0^{0}$, (c) $-15^{0}$. 
The temperatures obtained at different tool rake angle are shown in Fig.9. It can be seen that, with decrease in the tool rake angle, the workpiece surface temperature is almost unchanged. However, decreasing the tool rake angle leads to an increase of the PSZ and tool-chip interface temperature. It should be noticed that, the contact pressure and contact length could increase as the rake angle decreases [8]. Therefore, decreasing the rake angle enhances the friction between the tool and chip, which could result in an increase of the tool-chip interface temperature, since the temperature rise of the tool-chip interface is primarily due to the local heating caused by friction. In addition, the increase of friction increases the energy consumed in cutting, which could lead to an increase of cutting force, as shown in Fig.10.

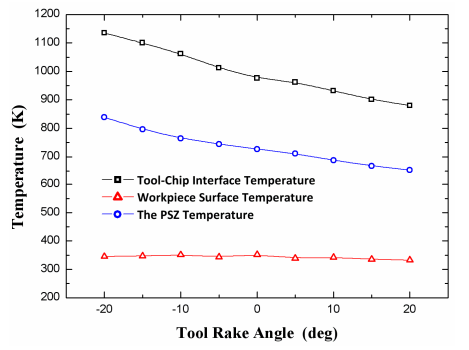

Fig.9 The temperatures versus tool rake angle

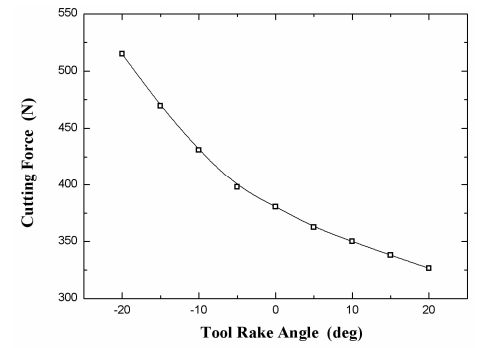

Fig.10 The cutting force versus tool rake angle.

\section{Conclusions}

In this work, the FEM was developed to simulate the orthogonal cutting of a TiAl6V4 alloy. The FEM was validated using experimental results and good agreement was obtained. The influence of cutting speed, uncut chip thickness and tool rake angle on the chip morphology, cutting force and temperature, was investigated. It is found that an increase in cutting speed and uncut chip thickness and a decrease in tool rake angle increases the PSZ temperature, which facilitates the saw-tooth chip formation. The results also reveal that, the Salomon's assumption is not valid for the tool-chip interface temperature, but could be true for the workpiece temperature. Furthermore, decreasing the uncut chip thickness leads to an increase of specific cutting energy, which could be attributed to a drop in the primary shear zone temperature with decrease in the uncut chip thickness.

\section{Acknowledgements}

This work was supported by the National Basic Research Program of China (Grant No: 2009CB724401), the Nature Science Foundation of China (Grants Nos.10725211, 11021262 and 11002144), and the National Natural Science Foundation of China-NSAF. Grant No: 10976100.

\section{References}

[1] K.M. Vernaza-Pefña, J.J. Mason and M. Li: Exp. Mech. Vol. 42 (2002), p. 221

[2] J. Barry, G. Byrne and D. Lennon: Int. J. Mach. Tools Manuf. Vol. 41 (2001), p. 1055

[3] W.N. Findley and R.M. Reed: ASME J. Eng. Ind. Vol. 85 (1963), p.49

[4] C. Hortig and B. Svendsen: J. Mater. Process. Technol. Vol. 186 (2007), p.66

[5] M. Calamaz, D. Coupard and F. Girot: Int. J. Mach. Tools Manuf. Vol. 48 (2008), p. 275

[6] C.J. Salomon, German Patent, 523594, 1931-04. (1931)

[7] M.C. Shaw: Metal Cutting Principles (Oxford University Press, UK 2005).

[8] S. Dechjarern: Asian Int. J. Sci. Technol. Prod. Manuf. Vol. 1 (2008), p.149. 
Materials Processing Technology, ICMPMT2011

10.4028/www.scientific.net/AMR.337

\section{Influence of Cutting Conditions on the Cutting Performance of TiAl6V4}

10.4028/www.scientific.net/AMR.337.387

\section{DOI References}

[2] J. Barry, G. Byrne and D. Lennon: Int. J. Mach. Tools Manuf. Vol. 41 (2001), p.1055. doi:10.1016/S0890-6955(00)00096-1

[3] W.N. Findley and R.M. Reed: ASME J. Eng. Ind. Vol. 85 (1963), p.49.

doi: $10.1115 / 1.3667593$

[4] C. Hortig and B. Svendsen: J. Mater. Process. Technol. Vol. 186 (2007), p.66.

doi:10.1016/j.jmatprotec.2006.12.018

[5] M. Calamaz, D. Coupard and F. Girot: Int. J. Mach. Tools Manuf. Vol. 48 (2008), p. 275.

doi:10.1016/j.ijmachtools.2007.10.014 